\title{
Transfert d'embryons chez les ovins et les caprins
}

\author{
P Brebion 1, G Baril 1, Y Cognié 1, JC Vallet 1,2 \\ 1 INRA physiologie de la reproduction et URA CNRS 1291, 37380 Nouzilly \\ 2 Procreatech-SA, Zl de la Centrale, 81400 Carmaux, France
}

(Reçu le 12 septembre 1991; accepté le 15 novembre 1991)

\begin{abstract}
Résumé - Les solutions techniques aux impératifs physiologiques qui régissent la réalisation du transfert d'embryons chez les petits ruminants sont en constante évolution. Nous en dressons une revue très sélective. Quelle qu'en soit la finalité, le transfert embryonnaire repose sur la capacité d'obtenir des donneuses une récolte suffisante d'embryons utilisables; la superovulation, première étape obligée, est induite à l'aide de préparations gonadotropes dont les plus appropriées sont les extraits hypophysaires, dits "FSH- $\mathrm{P}_{n} ; 3 \mathrm{j}$ de stimulation à activités FSH décroissante et $\mathrm{LH}$ croissante assurent en moyenne 11,1 (brebis) ou 14,2 (chèvre) ovulations avec respectivement $20 \%$ et $10 \%$ de réponses inférieures à 5 corps jaunes $(\mathrm{CJ})$. Chez la brebis, un prétraitement antigonadotrope préalable a permis d'accroître significativement la superovulation par élimination des nonréponses; chez la chèvre se pose le problème de la répétabilité de la stimulation par des hormones hétérologues, qui met en jeu la réponse immunitaire. Conséquence de la superovulation, l'altération de la survie et/ou de la remontée des spermatozoïdes dans le tractus femelle condamne l'IA exocervicale; chez la brebis si le dépôt in utero de $100 \times 10^{6}$ spermatozoïdes frais est effectué de façon standard $49 \mathrm{~h}$ après l'arrêt du traitement de synchronisation des chaleurs $77 \%$ des embryons collectés sont utilisables contre $87 \%$ si l'insémination est pratiquée «à la carte" $32 \mathrm{~h}$ après le début de l'œstrus ; chez la chèvre inséminée in utero avec $100 \times 10^{6} \mathrm{spz}$ congelés $45 \mathrm{~h}$ après l'arrêt du traitement progestagène, le taux de division moyen est de $71 \%$, mais il atteint $87 \%$ pour les femelles inséminées $15 \mathrm{~h}$ après leur pic de LH. Dans cette espèce la définition d'un moment d'IA standardisé mais adapté pour l'ensemble des donneuses suppose d'améliorer le groupement des ovulations. La collecte des morulas compactées/blastocystes a lieu 5-6 j (brebis) ou 6-7 j (chèvre) après la fécondation. L'infranchissabilite du cervix impose pour pratiquer le rétro-flushing des cornes une approche transpéritonéale. Après laparotomie, la réussite en première collecte est de $72-75 \%$ mais la répétabilité de l'intervention est faible; en revanche la méthode laparoscopique, un peu moins performante en première collecte (63\%), a été répétée avec succès jusqu'à 6-7 fois. La cryopréservation des embryons aux âges précisés fait appel à une technologie calquée sur le modèle bovin au moins pour la courbe de refroidissement. Avec 1,5 mol..$^{-1}$ d'éthylène-glycol comme cryoprotecteur et $20 \%$ de sérum de veau fœetal additionné au PBS (Phosphate Buffer Saline) enrichi (F1), nous obtenons 85 à $90 \%$ de réanimation, confirmés par un taux de survie après transfert de l'ordre de $65 \%$. Le transfert de 2 embryons par receveuse synchronisée est assuré par une technique laparoscopique légère, rapide et au moins aussi efficace que la méthode chirurgicale: avec $80 \%$ de fertilité et $80 \%$ de survie des embryons chez les receveuses fertiles, la survie globale des embryons transférés est de $65 \%$. Dépendante principalement de la productivité des traitements actuels en terme d'embryons utilisables, l'efficacité de la technologie du transfert embryonnaire permet donc d'espérer 4-5 produits (transfert direct) ou 3 produits (passage par la congélation) par donneuse traitée.
\end{abstract}


Summary - Embryo transfer in sheep and goats. In response to the physiological demands governing the feasibility of embryo transfer (ET) in small ruminants, the techniques involved are constantly improving, as we intend to show in the following selective review. Whatever the aim, ET is dependent on sufficient yields of viable embryos from donors. The first step in ET, superovulation, may be induced with various gonadotrophins, the most appropriate being the pituitary extracts known as FSH-P. Three days of stimulation with decreasing doses of follicle stimulating hormone (FSH) with increasing luteinizing hormone $(\mathrm{LH})$ content ensures mean ovulation rates of 11.1 in the ewe or 14.2 in the dairy goat, including $20 \%$ and $10 \%$ non-responses (< 5 corpora lutea $(C L)$ ), respectively. In the ewe, prior antigonadotrophic pretreatment results in a significant gain in ovulation rate due to the elimination of non-responses. In the goat, however, the responsiveness to repeated exogenous stimulation is impaired by the immune reaction. As a consequence of superovulation, there is a decrease in spermatozoal survival and/or transport within the donor genital tract which seems to condemn cervical artificial insemination. In the ewe, deposition of $100 \times 10^{6}$ fresh spermatozoa in utero $49 \mathrm{~h}$ after progestagen sponge removal results in the recovery of $77 \%$ viable embryos versus $87 \%$ when insemination occurs $32 \mathrm{~h}$ after the onset of oestrus. In the goat following deposition in utero of $100 \times 10^{6}$ frozen/ thawed spermatozoa $45 \mathrm{~h}$ after the end of progestagen treatment, the mean fertilization rate is $71 \%$ but it reaches $87 \%$ for females having their LH surge $15 \mathrm{~h}$ before the Al takes place. In this species, the definition of an efficient standardized timing for Al implies a better synchronisation of ovulations. Compacted morulae and/or blastocysts are recovered 5-6d (sheep) or 6-7d (goat) after fertilization. Because of the impenetrable cervix, retroflushing of the uterine horns with phosphate buffered saline (PBS) requires a transperitoneal approach. After laparotomy, first recovery rate reaches $72-75 \%$ but repeatability is limited, in contrast, laparoscopic recovery, although less efficient in first collection (63\%), has been successful up to 7 times. Cryopreservation of pre-hatched embryos is confined to bovine-inspired technology. Using $1.5 \mathrm{~mol}^{-1} \mathrm{r}^{-1}$ ethylene-glycol as a cryoprotectant and $20 \%$ FCS (foetal calf serum) added to PBS- $F_{1}$ (phosphate buffered saline- $F_{1}$ ) medium we obtained $85-90 \%$ reanimated embryos, as confirmed by $65 \%$ total survival following transfer. At least as effective as surgery but easier and quicker, laparoscopic transfer of 2 embryos in each synchronised recipient leads to $65 \%$ total survival ( $80 \%$ fertility and $80 \%$ surviving embryos in fertile recipients). In conclusion, ET technology which is mainly limited by the productivity of current treatments in term of usable embryos, allows 4-5 products (direct transfer) or 3 products (through deep frozen storage) per donor treatment.

\section{embryo transfer/sheep/goat/artificial insemination}

\section{INTRODUCTION}

L'objet de cette courte revue est de présenter un tableau sélectif de quelques unes des réponses techniques les mieux adaptées à ce jour aux conditions dictées par la physiologie, en identifiant pour chaque espèce les problèmes résiduels majeurs ainsi que les progrès les plus attendus à court terme.

En France en 1991, tandis que plus de 25000 transferts d'embryons sont annuellement pratiqués dans l'espèce bovine, cette activité reste marginale chez les petits ruminants, au moins quant à son application sur le terrain. Pourtant un intérêt potentiel existe, au même titre que chez les bovins, sous-tendu par les mêmes argumentaires génétique, commercial et sanitaire. II faut rechercher la raison de cette apparente stagnation dans le coût de production élevé d'un agneau/chevreau né de transfert embryonnaire par rapport à la valeur attribuée à l'animal. À niveaux de productivité (une moyenne de 4-5 embryons utilisables par donneuse traitée) et de fiabilité (une très large variabilité interindividuelle) équivalents aux résultats qui ont pu suffire à l'essor du transfert embryonnaire chez les bovins, cette technologie ne reste envisageable que dans de trop rares cas chez les ovins et caprins. Toute progression vers une meilleure productivité et surtout une moindre variabili- 
té de la réussite individuelle rendrait donc avantageux le recours au transfert embryonnaire chez les petits ruminants.

\section{TRANSFERT D'EMBRYONS : ÉTAPES TECHNIQUES, RENDEMENTS PARTIELS, RENDEMENT FINAL}

\begin{abstract}
À la différence de la procréation assistée dans l'espèce humaine, le transfert embryonnaire chez les animaux d'élevage n'a pas vocation thérapeutique; les traitements proposés, non personnalisables, doivent pouvoir être appliqués de façon standardisée à des lots de femelles, pour une efficacité moyenne dépassant nettement celle de la reproduction naturelle. Ceci implique toujours à la base l'obtention d'un nombre aussi élevé que possible d'embryons de qualité.
\end{abstract}

La figure 1 décrit les étapes techniques. Leurs rendements partiels $(R)$ sont successivement: la superovulation induite $\left(R_{0}=\right.$ $T_{0}=$ taux d'ovulation avec sa variabilité), la fécondation in vivo des ovocytes émis $\left(R_{1}=\right.$ taux de fécondation, ou, plus significatif, taux de viabilité des embryons), la collecte des embryons $\left(R_{2}=\right.$ taux de récupération par rapport au nombre d'ovulations), le transfert dans les receveuses $\left(R_{3}=\right.$ taux de survie des embryons transférés, combinant la fertilité après transfert et la prolificité des receveuses fertiles). Enfin, l'intérêt du transfert embryonnaire reposant largement sur la possibilité de dissocier dans l'espace et le temps les étapes de production et de transfert des embryons, la cryopréservation peut venir ajouter son rendement propre à la chaîne $\left(R_{4}=\right.$ taux de ranimation des embryons décongelés).

\section{SUPEROVULATION}

La superovulation des donneuses est induite au terme d'un traitement classique de synchronisation des chaleurs (par exemple par la méthode des éponges vaginales imprégnées de progestagène dans la formulation adaptée à l'espèce considérée). La fonction ovarienne peut être stimulée par une variété de préparations hormonales à activité gonadotrope (Chupin, 1988), comme eCG (equine chorionic gonadotrophin=pregnant mare serum gonadotrophin), HAP (horse anterior pituitary), FSH-P (follicle stimulating hormonepituitary) $\mathrm{pFSH}$ (porcine FSH), oFSH (ovine FSH), hMG (human menoposal gonadotrophin). Par delà la diversité des sources hormonales citées, il existe un relatif consensus sur les principes d'une stimulation de qualité:

- la durée de stimulation doit avoisiner celle de la croissance folliculaire terminale (ici 3 j);

- l'activité FSH doit nettement prédominer sur celle de la LH contaminante en début de traitement (le seuil de tolérance semble varier selon le génotype; chez la brebis Lacaune un rapport $\mathrm{FSH} / \mathrm{LH}<2$ affecte la qualité embryonnaire); ceci condamne notamment l'usage de eCG, molécule dont l'activité intrinsèque $\mathrm{LH}$ est environ 4 fois supérieure à l'activité $\mathrm{FSH}$; un enrichissement en LH semble en revanche nécessaire en fin de traitement (FSH/LH $<0,4$ ), conditionnant l'induction des ovulations (Cognié et al, 1986). Deux traitements types utilisant pFSH sont détaillés à titre d'exemple figure 2.

Même si des améliorations de ces conditions de stimulation sont encore possibles, notamment par l'utilisation des facteurs de croissance dans la maturation folliculaire terminale, le type de traitement décrit ci-dessus représente un optimum relatif, puisqu'il permet la pleine expression de l'état de l'ovaire au moment de la première dose stimulante.

Cependant les résultats des traitements, même les plus évolués, désignent 


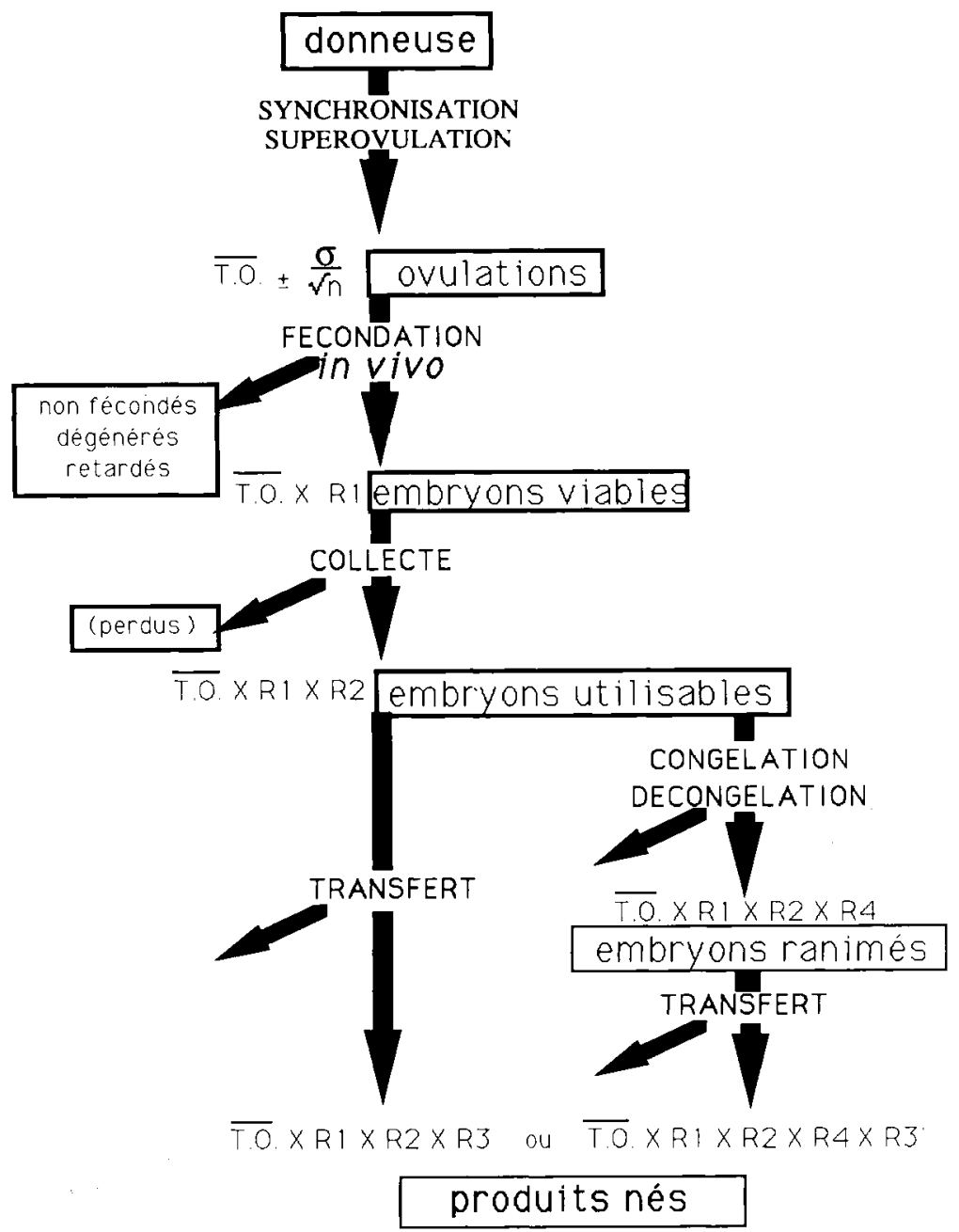

Fig.1. Transfert d'embryons : enchainement des étapes, combinaison des rendements.

encore la superovulation comme l'étape la plus limitante de la production d'embryons. En effet (fig 3), autour de réponses moyennes de $11 \mathrm{CJ}$ (brebis laitière) ou 14 CJ (chèvre laitière) la variabilité interindividuelle est excessivement élevée, incluant $20 \%$ de non-réponses ( $<5$ ovulations).
Cette variabilité reflète très exactement la structure de la population folliculaire de l'ovaire stimulé. Chez la brebis superovulée avec pFSH le TO est positivement corrélé au nombre de follicules de 1-2 $\mathrm{mm}$ présents au début de la stimulation. Un prétraitement antigonadotrope par des 

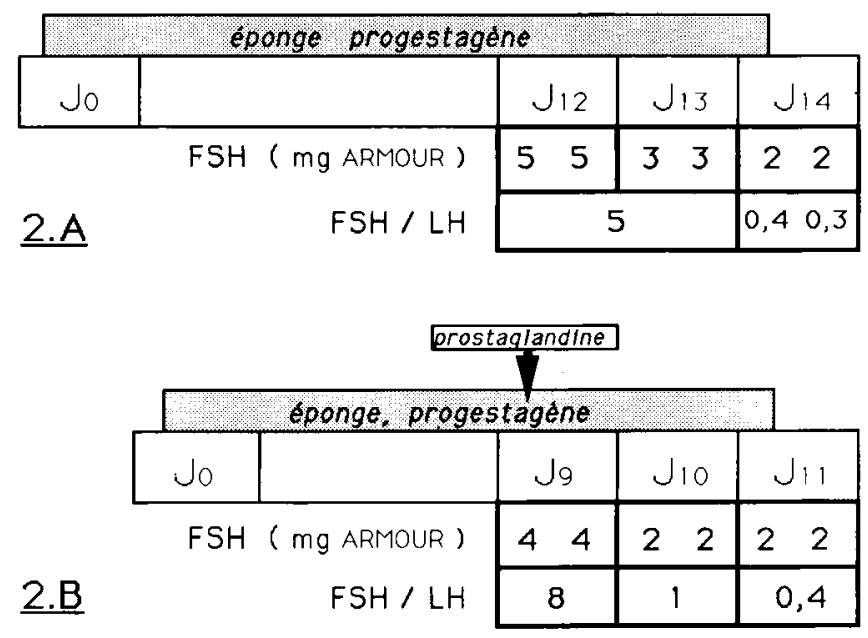

Fig 2. Traitements de superovulation applicables à la brebis (2A) ou à la chèvre (2B).
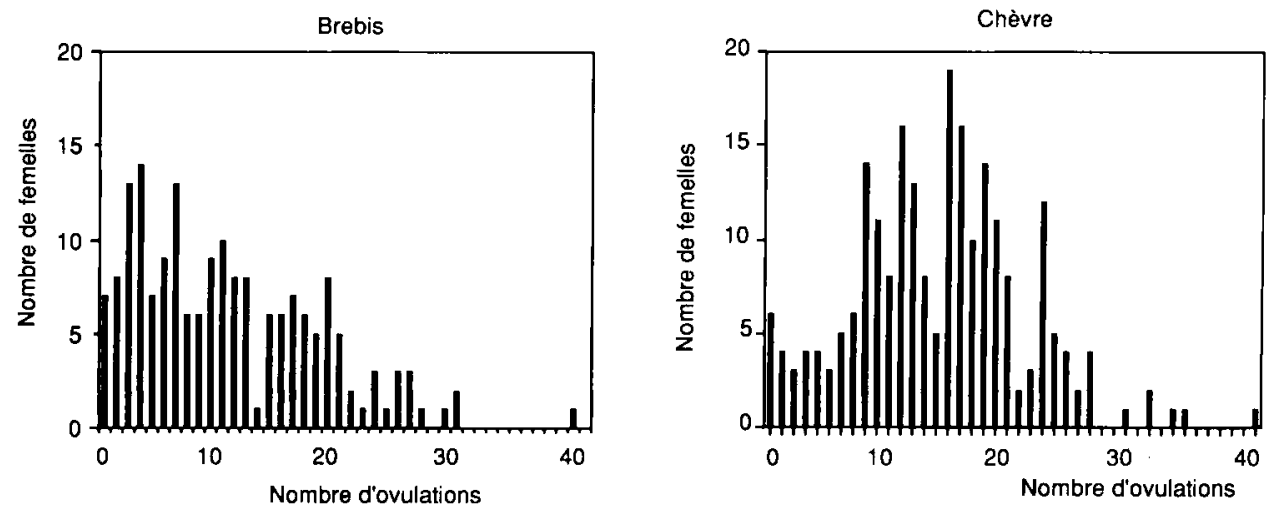

Fig 3. Répartition des femelles en fonction du nombre d'ovulations; brebis : après $20 \mathrm{mg}$ de FSH ( $n=$ 198), chèvre : après $16 \mathrm{mg}$ de FSH $(n=226)$.

agonistes ou un antagoniste (tableau I) du $\mathrm{GnRH}$ a permis d'enrichir cette classe de follicules cibles, doublant le nombre moyen d'ovulations par élimination des faibles réponses (Brebion et Cognié, 1989). Le même principe est à l'étude chez la chèvre.

En ce qui concerne la répétabilité de la superovulation, la situation change avec l'espèce considérée. Chez la brebis de gé- 
Tableau I. Effets de $11 \mathrm{j}$ de traitement par un antagoniste du GnRH sur l'ovaire et la superovulation consécutive, chez la brebis Lacaune. vation de la liaison plasmatique de $\mathrm{pFSH}$, indicatrice de la présence d'anticorps anti FSH (Rémy et al, 1992).

\section{FÉCONDATION}

La fécondation in vivo des ovocytes de fe$\mathrm{Nb}$ follicules

$\begin{array}{lcc}\begin{array}{l}\text { de diamètre 1-2 } \mathrm{mm} \\ \text { avant FSH }\end{array} & 24,7 & 12,5 \\ \begin{array}{l}\text { Nb d'ovulations ** } \\ \text { après FSH } \\ \text { (m } \pm \text { sem) }\end{array} & 19,2 \pm 3,4 & 9,9 \pm 1,6 \\ \begin{array}{l}\text { \% de collecte } \\ \text { des embryons }\end{array} & 67,5 & 66,0 \\ \begin{array}{l}\text { Nb d'embryons } \\ \text { viables }(\%)\end{array} & 10,6(81) & 6,1(92)\end{array}$

notype croise hyperprolifique Booroola $x$ Romanov traitée $6-7$ fois à intervalles de 60 j par $20 \mathrm{mg} \mathrm{pFSH}$, aucune diminution significative de la réponse n'est observée (Brebion et al, 1990). En revanche, chez des chèvres laitières soumises à 5 traitements successifs par $16 \mathrm{mg}$ pFSH à intervalles de $50 \mathrm{j}$, l'efficacité diminue fortement avec le rang de traitement, les nonréponses ayant pu être reliées à une élé- melles superovulées pose des difficultés particulières (Armstrong et Evans, 1983), liées non au traitement en lui-même (sauf utilisation de PMSG), mais dépendantes de la réponse ovarienne. Le taux de fécondation après IA classique est corrélé négativement au taux d'ovulation (chèvre: Baril et al, 1989). Une altération massive des conditions de survie et de transit des spermatozoïdes impose de déposer ceux-ci le plus près possible du site de fécondation. Chez la brebis (tableau II) et la chèvre superovulées avec pFSH, l'insémination intra-utérine (IAU) sous contrôle laparoscopique permet d'obtenir un pourcentage élevé d'embryons viables quel que soit le niveau de réponse. Chez la brebis inséminée in utero avec $100.10^{6} \mathrm{spz}$ consenvés à $+15^{\circ} \mathrm{C}$, la qualité embryonnaire est maximale si I'IAU est réalisée $2-6 \mathrm{~h}$ après l'ovulation $(87 \%$ de fécondation alors que la moyenne générale est de $70 \%$ par cette technique) $49 \mathrm{~h}$ après la fin du traitement

Tableau II. Efficacités comparées de I'IA intra-utérine et de I'IA cervicale pour la production d'embryons chez la brebis.

IA exocervicale

$48+60$

$2 \times 400$

Nombre de spz (en 106)

Embryons

Fécondés (\%)

Viables

$\%$ total

Nb par femelle collectée

$\mathrm{Nb}$ par femelle traitée
IA intra-utérine
$1 \times 80$

93

$\begin{array}{lll}45 & 44 & 86\end{array}$

$\begin{array}{lll}3,5 & 3,8 & 6,7\end{array}$ 
progestatif (77\% d'embryons viables). Chez la chèvre inséminée in utero avec $100.10^{6} \mathrm{spz}$ congelés, la meilleure qualité est obtenue pour les femelles inséminées $16 \mathrm{~h}$ après leur pic de $\mathrm{LH}$, soit environ $6 \mathrm{~h}$ avant le début des ovulations ( $87 \%$ de fécondation, alors que la moyenne générale est de $70 \%$ par cette technique). La faisabilité de l'IAU au moment optimal pour une majorité de femelles traitées conjointement dépend du degré de synchronisation des ovulations à la suite du traitement pratiqué. Quatre vingt cinq pour cent des brebis traitées ovulent pendant une période de $20 \mathrm{~h}$, contre $40 \mathrm{~h}$ chez la chèvre. Pour cette raison, la qualité embryonnaire est le problème majeur dans cette dernière espèce. En attendant de savoir regrouper les ovulations sans compromettre la maturation finale de l'ovocyte, il est indispensable de pratiquer I'IAU par rapport au pic de $\mathrm{LH}$ (précis mais difficile à mettre en œuvre) ou, à défaut, 20-24 $\mathrm{h}$ après le début des chaleurs (pratique mais moins précis).

\section{COĹLECTE DES EMBRYONS}

Les embryons sont généralement recueillis aux stades "morula compactée" à ublastocyste» (80-300 cellules) correspondant à J6-J7 chez la brebis et J6,5-J7,5 chez la chèvre, $\mathrm{J} 1$ étant le jour de la fécondation.

Chez l'une et l'autre espèce, la difficulté de franchissement du cervix (Kraemer, 1989) impose raisonnablement une approche transpéritonéale. De nombreuses variantes techniques sont possibles pour parvenir à perfuser chaque corne utérine avec $40 \mathrm{ml}$ de milieu PBS dans le sens antégrade ou rétrograde. Le véritable choix qui s'offre au praticien porte entre la laparotomie classique et une technique moins invasive sous contrôle laparoscopique:

- la laparotomie médioventrale permet de collecter aisément $72 \%$ d'embryons (taux de collecte à J6) par rapport aux CJ. Toutefois la présence d'adhérences postopératoires à partir de la deuxième collecte, peut compromettre tant la fertilité que la réussite des collectes ultérieures;

- la collecte laparoscopique (Vallet, 1987; Scudamore et Robinson, 1990), de maîtrise plus délicate, offre un rendement moyen de $62 \%$; cette technique a été développée pour son innocuité. De fait, elle n'affecte pas la fertilité des sujets, et a pu être répétée jusqu'à 7 fois sans perte d'efficacité.

\section{CRYOPRÉSERVATION}

On ne congèle actuellement avec succès que les morula compactées et les blastocystes selon une technologie dérivée du modèle bovin. Les variantes concement le choix du cryoprotecteur. Chez la brebis et la chèvre l'éthylène glycol 1,5 mol.t-1 ajouté au milieu $F_{1}$ peut être retenu. L'avantage de fractionner en 3 étapes l'addition du cryoprotecteur n'est pas net. La courbe de refroidissement comporte un palier à $-7^{\circ} \mathrm{C}$ puis une pente de $-0,3 \% \mathrm{~min}$ jusqu'à $-30^{\circ} \mathrm{C}$, où a lieu l'immersion dans l'azote liquide $\left(-196^{\circ} \mathrm{C}\right)$. Si l'on part d'embryons exempts de toute anomalie morphologique (classe A) on peut prévoir un taux de ranimation moyen de $70 \%$, dont la variabilité (50-90\%) rappelle que le jugement visuel des embryons donne une estimation très imparfaite de leur viabilité réelle.

L'intérêt de pouvoir congeler des embryons plus jeunes serait grand, car on sait que le rendement d'une collecte plus précoce est supérieur, particulièrement dans l'oviducte à J3,5 (Folch et al, 1991).

\section{TRANSFERT}

La laparotomie classique peut être aujourd'hui avantageusement remplacée par 
Tableau III. Fertilité, en \% de fernelles mettant bas (nombre de femelles receveuses), après transfert laparoscopique ou chirurgical chez la brebis et chez la chèvre.

\begin{tabular}{llllll}
\hline & \multicolumn{2}{c}{ Brebis } & & \multicolumn{2}{c}{ Chèvre } \\
\cline { 2 - 3 } \cline { 5 - 6 } & $\begin{array}{c}\text { Embryons } \\
\text { frais }\end{array}$ & $\begin{array}{l}\text { Embryons } \\
\text { congelés }\end{array}$ & & $\begin{array}{c}\text { Embryons } \\
\text { frais }\end{array}$ & $\begin{array}{c}\text { Embryons } \\
\text { congelés }\end{array}$ \\
\hline Transfert laparoscopique & $80(46)$ & $90(20)$ & & $76(25)$ & $75(12)$ \\
Transfert chirurgical & $88(25)$ & $45(11)$ & & $64(25)$ & $89(9)$ \\
\hline
\end{tabular}

une technique laparoscopique au moins aussi efficace et bien mieux adaptée aux conditions de terrain (Vallet et al, 1990). À la suite au transfert de 2 embryons frais par receveuse synchronisée la fertilité est comprise entre 70 et $80 \%$ (tableau III); la survie des embryons chez les receveuses fertiles étant de $80 \%$, la survie moyenne de l'embryon frais transféré est de $65 \%$. Pour un embryon décongelé et jugé viable, la survie globale est de $50-55 \%$.

\section{Conclusion : Rendement final du transfert embryonnaire}

La figure 4 présente 4 versions aujourd'hui possibles du transfert embryonnaire chez la brebis ou chez la chèvre, qui intègrent plus ou moins les nouvelles connaissances relatives à la superovulation et aux conditions d'insémination. La démonstration de la possibilité d'une préparation de l'ovaire réduisant l'importante variabilité de la superovulation est intéressante dans ces 2 espèces, mais pourrait l'être également chez les bovins où ce problème n'est pas résolu actuellement. Chez les petits ruminants, l'émergence de cette nouvelle génération de traitements caractérisés par un contrôle total des phénomènes, depuis la préparation ovarienne jusqu'au déclenchement des ovulations est une étape im-

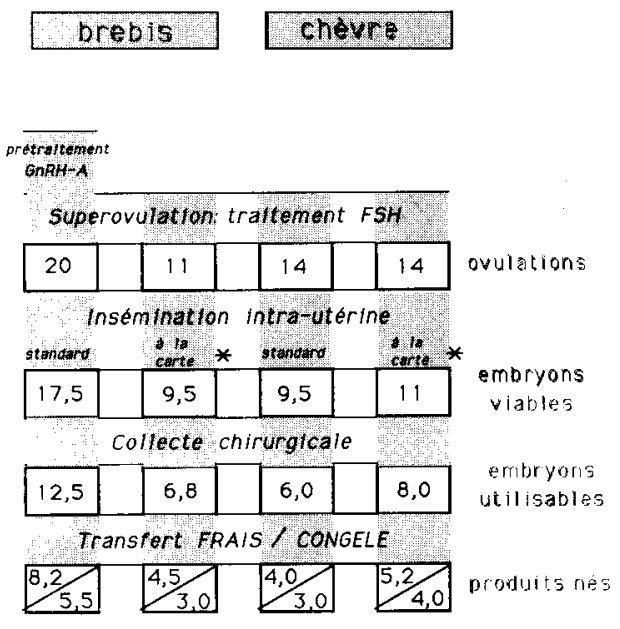

Fig 4. Nouvelles possibilités pour le transfert embryonnaire chez la brebis et la chèvre. "IA «à la carte $=27$ heures après le pic de LH (brebis, sperme frais) ou 15 heures après le pic de LH (chèvre, sperme congelé).

portante pour le développement du transfert embryonnaire. Au delà d'un accroissement de la productivité moyenne (plus de 10 embryons utilisables par brebis prétraitée), l'apport majeur sera la réduction du pourcentage d'échecs individuels encore aujourd'hui excessif dans toutes les espèces. 


\section{RÉFÉRENCES}

Armstrong DT, Evans G (1983) Factors influencing success of embryo transfer in sheep and goats. Theriogenology 19, 31-42

Baril G, Casamitjana P, Perrin J, Vallet JC (1989) Embryo production, freezing and transfer in Angora, Alpine and Saanen goats. Zuchthygiene (Berl) 24, 101-115

Brebion $P$, Cognié $Y$ (1989) Increased superovulation in the ewe following 14 days of GnRHagonist pretreatment. AETE, $5^{e}$ Coll Sci, p 106

Brebion $P$, Beckers JF, Guérin $Y$, Boomarov (1990) High performance of Booroola $x$ Romanov ewes as permanent embryo donors. In: Colloq INRA, major genes for reproduction in sheep, 171-174

Chupin D (1988) Superovulation par PMSG ou FSH pour le transfert embryonnaire. Coll Soc Fr Études Fertil 26, 213-232

Cognié $Y$, Chupin D, Saumande $J(1986)$ The effect of modifying the $\mathrm{FSH} / \mathrm{LH}$ ratio during the superovulatory treatment in ewes. Theriogenology 25,148
Folch J, Cocero MJ, Ramon JP, Fernadez-Arias A, Alabart JL (1991) Embryo recovery in the oviduct improves efficiency of superovulation in the ewe. AETE 70 Coll Sci, $144 p$

Kraemer DC (1989) Embryo collection and transfer in small ruminants. Theriogenology 31, 141-148

Rémy B, Baril G, Vallet JC, Dufour R, Chouvet C, Saumande J, Chupin D, Bechers JF (1992) Are antibodies responsible for a decreased superovulatory response in goats which have been treated repeatedly with porcine follicle-stimulating hormone? Theriogenology 36, 389-399

Scudamore CL, Robinson JJ (1990) Laparoscopic embryo recovery and transfer in sheep. Proc Soc study anim breeding; 1988-1989, 27-35

Vallet JC (1987) Feasability and repeatability of embryo recoveries from dairy goats under laparoscopy. AETE, $3^{\circ}$ Coll Sci, $159 \mathrm{p}$

Vallet JC, Baril, G, Loysel C (1990) Surgical or laparoscopic embryo transfer in goats. AETE, $5^{\circ}$ Colloq Sci, $186 \mathrm{p}$ 